lie for tragedies where there has been no outreach, and previous carers may not even know about them?

Conclusions from the three-year study await our final report this year. They will be based on the clinical and social outcomes, users' (patients and relatives) satisfaction, risk assessment, and an economic analysis.

Research and Development for Psychiatry

Matt Muluen 134/138 Borough High Street

London SEI ILB

Institute of Psychiatry

De Crespigny Park

London SE5 8AF

\section{Enhancement of recovery from psychiatric illness by methylfolate}

SIR: We are grateful to Dr Procter (Journal, August 1991, 159, 271-272), Dr Anderson et al (Journal, January 1992, 160, 130) and Drs Wing \& Lee (Journal, May 1992, 160, 714-715) for their interest in our study reporting enhancement of recovery from psychiatric illness by methylfolate (Godfrey et al, 1990). Procter is sufficiently impressed by this and earlier literature to doubt the need for further controlled trials of treatment of folate deficiency in psychiatric patients. Anderson et al and Wing \& Lee have some reservations about some aspects of the design of our trial, while at the same time stressing the importance of the folate-mental disorder connection and drawing attention to their own interesting contributions to the subject.

To deal first with the design features of our methylfolate trial, we do not agree that it was inappropriate to use the Hamilton Rating Scale for Depression (HRSD) in schizophrenic patients. The HRSD is as valid for depression as a symptom in other psychiatric disorders as it is in depressive illness. Nor did we find the inconsistencies between the HRSD and Beck scores surprising. Many of our patients were psychotic, and self-report in such patients, many of whom are without insight, is bound to be unreliable. The object of our study was to evaluate long-term (six months) clinical outcome, and the use of clinical outcome scales in such a context is well established in psychiatric research. We do not agree that it is difficult to distinguish between residual symptoms and pronounced impairment, or between full social recovery and no or partial social recovery. The absence of baseline scores was dictated by the fact that the point of entry to the trial was the finding of a low or borderline red cell folate level, at which point many of the patients were already on standard medication. We were studying in a placebocontrolled manner the outcome of an adjunct to treatment and we had no wish, nor would it have been practical, to influence the normal treatment that these patients were otherwise receiving. We cannot exclude the possibility of baseline differences occurring due to chance, but nor have we any reason to believe that such differences exist.

We agree with Procter and have stressed for many years (Carney, 1967; Reynolds, 1967) that our observations are relevant to other psychiatric diagnostic categories. The influence of folate treatment in different mental disorders may be mediated by an effect on mood, drive, initiative and sociability (ultimately cognition), as may also be the case for S-adenosylmethionine, a metabolite closely related to folate in methylation processes within the nervous system (Reynolds et al, 1984).

Procter and Anderson et al discuss possible connections with monoamine and biopterin metabolism, which we have emphasised since our earliest studies of folate (Reynolds et al, 1970, 1984). In a recent study of 34 patients with a DSM-III diagnosis of depression (Bottiglieri et al, 1992) we found significant positive correlations between red cell folate and cerebrospinal fluid 5-hydroxyindoleacetic acid (CSF 5-HIAA), homovanillic acid (HVA), and tetrahydrobiopterin (BH4). As expected, there were also significant correlations between the latter three CSF metabolites. These observations further support the links between folate and monoamine theories of affective disorder. This and other recent studies (Carney et al, 1990) also emphasise the importance of studying the more stable red cell folate in psychiatric disorders and we would encourage Anderson et al and Wing \& Lee to do so in their own studies.

Bottiglieri, T., Hyland, K., LAundy, M., et al (1992) Folate deficiency, biopterin and monoamine metabolism in depression. Psychological Medicine (in press).

CARneY, M. W. P. (1967) Serum folate values in 423 psychiatric patients. British Medical Journal, iv, 512-516.

- Chary, T. K. N., LAUNDY, M., et al (1990) Red cell folate concentrations in psychiatric patients. Journal of Affective Disorders, 19, 207-213.

Godfrey, P. S. A., TOONe, B. K., Carney, M. W. P., et al (1990) Enhancement of recovery from psychiatric illness by methylfolate. Lancet, 336, 392-395.

REYNolds, E. H. (1967) Efiects of folic acid on the mental state and fit frequency of drug-treated epileptic patients. Lancet, $i$, 1086-1088.

, Preece, J. M., Bailey, J., el al (1970) Folate deficiency in depressive illness. British Journal of Psychiatry, 117, 287-292. 
Reynolds E. H., Carney, M. W. P. \& ToOne, B. K. (1984) Methylation and mood. Lancet, ii, 196-198.

P. GODFREY

R. CREllin

Department of Psychiatry

King's College Hospital

London SE5 9RS

Department of Psychiatry

Northwick Park Hospital

Harrow, Middlesex

Department of Haematology

Northwick Park Hospital

Department of Neurology

Institute of Psychiatry and

King's College Hospital

London SE5 8AF

Schizophrenia following prenatal exposure to infuenza epidemics between 1939 and 1960

SIR: Professor Murray and his colleagues are to be congratulated upon their careful demonstration of an association between the timing of influenza epidemic deaths and the rate of births of individuals who later develop schizophrenia (Journal, April 1992, 160, 461-466). They take this as further support for their proposition that schizophrenia is best viewed as a neurodevelopmental disorder in which prenatal damage arising from viral infection may well play a part in some cases. As they point out, this idea is compatible with their evidence and that of other studies. However, there is at least one alternative explanation. The observed association could arise if those liable to future schizophrenia were more likely to survive prenatal insult such as maternal influenza than those who are not so liable. Were this liability to schizophrenia and to survival of prenatal insult to be genetically determined, it might also explain the persistence of the relevant gene in the population by providing some selective advantage to balance the disadvantage of the low fertility of schizophrenic people.

Department of Psychiatry

R. L. PALMER

University of Leicester

Leicester General Hospital

Gwendolen Road

Leicester LE5 4PW

\section{Psychotherapy in non-Western cultures}

SIR: Long-term psychotherapy is difficult to implement in non-Western countries. Patients who seek help expect immediate relief in a short time. The stigma of mental illness is very powerful, especially in close religious communities. In addition, psychiatry is considered suspect, as a challenge to the existence of God, and many believe that turning to a psychiatrist is an act of weak faith, as it is God who heals all ills (Peteet, 1981).

Cross-cultural psychiatry tries to understand the complaints of people from different cultures in order to verify or modify existing models of mental illness (Kleinman, 1987).

Short-term psychotherapy is used to emphasise problem-solving, with a limited dynamic change. The anxiety generated during the interview may be used as a tool in assisting the patient to change his maladaptive behaviour and to attain a state of improved emotional functioning. This type of psychotherapy is offered to patients with average intelligence, who have shown some affect during interview and are motivated to work hard with their therapists. Therapists encourage the establishment of rapport with their patients, creating a therapeutic alliance by using positive transference feelings and concentrating on the unresolved emotional conflicts underlying patients' symptoms.

Brief therapy and crisis intervention should be offered to seriously disturbed patients who have recently decompensated. The aim here is to decrease or eliminate anxiety, by the use of supportive techniques, such as reassurance or environmental manipulation, with or without medication. The patients need guidance to come to grips with their problems and conflicts.

The therapist has to see the patient one to three times a week, in average 30-40-minute sessions for a period of three to six months. The therapist has to express warmth and caring for the patient. The language is one of understanding, love, insight, and human involvement (Truax, 1964).

He has to show the patient that he is eager to help, and allow him to talk freely without interruption. Appropriate medication is used when necessary. Political and religious views are separately handled. The therapist has to approach the patient through the social organisation of his religious group and not to be influenced by his personal attitude towards religion or politics.

KLeinman, A. (1987) Anthropology and psychiatry: the role of culture in cross-cultural research on illness. British Journal of Psychiatry, 151, 447-454. 